\title{
Path towards efficient paediatric formulation development based on partnering with clinical pharmacologists and clinicians, a c4c expert group White paper.
}

Jennifer Walsh ${ }^{1}$, Daniel Schaufelberger ${ }^{2}$, Sonia Lurian ${ }^{3}$, Sandra Klein ${ }^{4}$, Hannah Batchelor ${ }^{5}$, Roy Turner ${ }^{6}$, Sveinbjörn Gizurarson ${ }^{7}$, Luigi Boltri ${ }^{8}$, Elisa Alessandrini ${ }^{9}$, and Catherine Tuleu ${ }^{10}$

${ }^{1}$ Jenny Walsh Consulting Ltd

${ }^{2}$ 2. Schaufelberger Consulting LLC

${ }^{3}$ Iuliu Haţieganu University of Medicine and Pharmacy

${ }^{4}$ Ernst-Moritz-Arndt Universitat Greifswald Mathematisch-Naturwissenschaftliche Fakultat

${ }^{5}$ University of Strathclyde

${ }^{6}$ Idorsia Pharmaceuticals Ltd

${ }^{7}$ University of Iceland

${ }^{8}$ Adare Pharmaceuticals

${ }^{9}$ UCL School of Pharmacy

${ }^{10}$ University College London School of Pharmacy

April 22, 2021

\begin{abstract}
Improved global access to novel age-appropriate formulations for paediatric subsets, either of new chemical entities or existing drugs, is a priority to ensure that medicines meet the needs of these patients. However, despite regulatory incentives, the introduction to the market of paediatric formulations still lags behind adult products. This is mainly caused by additional complexities associated with the development of acceptable age-appropriate paediatric medicines. This position paper proposes the use of a paediatric Quality Target Product Profile (pQTPP) as an efficient tool to facilitate early planning and decision making during the children-centric formulation design for new chemical entities, or to repurpose/reformulate off-patent drugs. Essential key attributes of a paediatric formulation are suggested and described. Moreover, greater collaboration between formulation experts and clinical colleagues, including healthcare professionals, is advocated to lead to safe and effective, ageappropriate medicinal products. Acceptability testing should be a secondary endpoint in paediatric clinical trials to ensure post-marketing adherence is not compromised by a lack of acceptability. Not knowing the indications and the related age groups and potential dosing regimens early enough is still a major hurdle for efficient paediatric formulation development; however the proposed PQTPP could be a valuable collaborative tool for planning and decision making to expedite paediatric product development.
\end{abstract}

\section{Hosted file}

BJCP manuscript C4C WP4 20210331 JW CT EA.pdf available at https://authorea.com/users/ 409462/articles/519138-path-towards-efficient-paediatric-formulation-development-basedon-partnering-with-clinical-pharmacologists-and-clinicians-a-c4c-expert-group-whitepaper 